\title{
Infection and dissemination of two dengue type- 2 viruses isolated from patients exhibiting different disease severity in orally infected Aedes aegypti from different geographic origin
}

\author{
Ronald Enrique Morales ${ }^{1}$, Kouichi Morita ${ }^{2)}$, Yuki Eshita ${ }^{3)}$, \\ Yoshio TsUdA ${ }^{1)}$, Toshihide FUKUmA ${ }^{4}$ and Masahiro TAKAgI ${ }^{1)}$ \\ 1) Department of Vector Ecology \& Environment, Institute of Tropical Medicine, Nagasaki University, \\ Nagasaki, 852-8523 Japan \\ 2) Department of Virology, Institute of Tropical Medicine, Nagasaki University, Nagasaki, 852-8523 Japan \\ 3) Department of Infectious Disease Control, Oita Medical University, Oita, 879-5593 Japan \\ 4) Department of Parasitology, Kurume University School of Medicine, Kurume, 830-0001 Japan
}

(Received: October 18, 2001; Accepted: December 25, 2001)

Key words: infection and dissemination, Aedes aegypti, geographic strain, dengue type- 2 virus, disease severity

\begin{abstract}
The capacity for oral infection and dissemination of two dengue type2 viruses, ThNH7/93 and ThNHpll/93, isolated from patients exhibiting different disease severity, dengue shock syndrome (DSS) and classical dengue fever (DF), respectively, was compared in two strains of Aedes aegypti from different geographic origin. The capacity for oral infection and dissemination to the head tissues of the isolate ThNH7/93 (DSS) was significantly higher in the Ae. aegypti strain from Nakhon Phanom, Thailand, than that in the strain from Escuintla, Guatemala, $\left(P<0.05, \chi^{2}=\right.$ $6.833, \mathrm{df}=1)$, whereas for the isolate ThNHpll/93 (DF) the capacity was not significantly different between the Ae. aegypti strains $\left(P>0.05, \chi^{2}=0.557, \mathrm{df}=1\right)$. The Ae. aegypti strain from the location where the viruses were isolated (Nakhon Phanom, Thailand) showed significantly higher susceptibility to the isolate ThNH7/93 strain than to the isolate ThNHpll $/ 93\left(P<0.05, \chi^{2}=13.283, \mathrm{df}=1\right)$, while the susceptibility to both isolate virus strains was not significantly different in the Ae. aegypti strain from Guatemala ( $P$ $\left.>0.05, \chi^{2}=0.049, \mathrm{df}=1\right)$. These results suggested that the efficacy of dengue virus circulation was likely to vary according to combination of the virus strains and origin of the vector mosquitoes.
\end{abstract}

\section{INTRODUCTION}

Dengue (DEN) viruses with 4 different serotypes, causing dengue fever (DF), dengue haemorrhagic fever (DHF), and dengue shock syndrome (DSS) are a medically important arthropod-borne virus affecting humans in terms of morbidity (Monath and Heinz, 1996; WHO, 2000). $\mathrm{DF} / \mathrm{DHF}$ and DSS have re-emerged mainly in tropical and subtropical regions, and DHF has become the leading cause of death and hospitalization among children in some Southeast Asian countries during the last two decades (Gubler and Clark, 1995; Monath, 1994).

Inter-population variation in vector competence of mosquito species for arbovirus has been well documented: $A e$. albopictus and chikungunya virus (Tesh et al., 1976), Ae. aegypti and yellow fever (Aitken et al., 1977), and Ae. triseriatus and La Crosse virus (Grimstand et al., 1977). As for flavivirus, significant variation in oral susceptibility among geographic strains of Ae. albopictus and Ae. aegypti for the 4 dengue serotypes has been reported 
(Gubler and Rosen, 1976; Gubler et al., 1979; Rosen et al., 1985; Tardieux et al., 1990). Differences have also been reported among Ae. albopictus strains in the rates of dengue virus infection, dissemination, and transmission (Boromisa et al., 1987). However, the mechanisms responsible for intra and interspecific variation in the ability of Aedes subgenus Stegomyia mosquitoes for infection and transmission of dengue (DEN) viruses are not well understood. Some previous works proposed that it was genetically controlled (Gubler and Rosen, 1976; Gubler et al., 1979), while others considered that the variability of the oral infection rate was attributed to the mesenteronal barrier, since no significant differences were noted when several strains of Ae. aegypti and Ae. albopictus were infected by parenteral inoculation (Rosen et al., 1985).

Pandy and Igarashi (2000) in a molecular study on dengue type- 2 viruses has classified the virus of the Southeast Asian genotype isolated from patients exhibiting different disease severity into 3 subtypes according to non-synonymous amino acid replacements. In parallel, in vitro experiments on growth rate of dengue type-2 viruses have shown that representative strains from each of the 3 different amino acid sequence subtypes of the virus showed different infection rates to a primary culture of human peripheral blood leukocytes; hence, it was concluded that the infection rate correlated with the disease severity (Mangada and Igarashi, 1998). In addition, Morens et al. (1991) has reported that the observed cytopathic effects in C6/36 cells correlated with disease severity.

Despite all these observations, there was no document on the rates of susceptibility of $A$ e. aegypti to oral infection with virus isolated from patients exhibiting different disease severity. Thus, in this study the infection and dissemination rates of two strains (ThNH7/93 and ThNHpll/93) of dengue type- 2 virus isolated from patients exhibiting different disease severity during the same epidemic season in the same epidemic area were evaluated in orally infected $A e$. aegypti from different geographic origin.

The aim of this study was to evaluate the oral infection and dissemination of two dengue type- 2 virus isolated from patients exhibiting different disease severity in $A e$. aegypti of different geographic origin, one of which was originated from Guatemala where the dengue type- 2 virus of Southeast Asian genotype had not been reported to be prevalent.

\section{Materials AND Methods}

\section{Mosquito strains}

Two geographically different $A e$. aegypti strains were used in this study. The dengue epidemiological background of the site where the mosquitoes were collected is presented in Table 1 . The strain from Thailand was derived from eggs oviposited by field-collected adults, while the strain from Guatemala was derived from field-collected eggs. The mosquitoes were reared in plastic pans $(33 \mathrm{~cm} \times 25 \mathrm{~cm} \times 11$ $\mathrm{cm}$ high approximately) containing $3 l$ of

Table 1. Geographic origin and dengue epidemiology information of the area where the Aedes aegypti strains were collected.

\begin{tabular}{|c|c|c|}
\hline Geographic origin & Year of colonization & Dengue epidemiology background \\
\hline Nakhong Phanon Thailand & January 1997 & $\begin{array}{l}\text { Dengue endemic area, with previous report of } \\
\text { DSS, DHF cases. }\end{array}$ \\
\hline Escuintla $^{a}$ Guatemala & December 1991 & $\begin{array}{l}\text { Dengue endemic area with a recent report of } \\
\text { DHF cases (Sept. 2000) }\end{array}$ \\
\hline
\end{tabular}

a Supplied by Dr. I. Tanaka from Department of Environmental Biology, Japan Environmental Sanitation Center. 
Table 2. Comparison of the dengue type- 2 isolates from Thailand.

\begin{tabular}{|c|c|c|}
\hline & \multicolumn{2}{|c|}{ Isolate Name } \\
\hline & ThNH7/93 & ThNHp11/93 \\
\hline Clinical severity ${ }^{a}$ & DSS & DF \\
\hline Serological response $\mathrm{b}^{\mathrm{b}}$ & Secondary & Primary \\
\hline $\begin{array}{l}\text { Average Plaque size } \\
(\mathrm{mm})(\mathrm{LLC}-\mathrm{MK} 2)^{c}\end{array}$ & 1.67 & 0.82 \\
\hline AA subtype ${ }^{d}$ & I & III \\
\hline
\end{tabular}

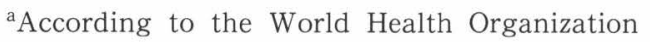
criteria. ${ }^{\mathrm{b}}$ IgG-ELISA assay results. ${ }^{\mathrm{c}}$ Mangada and Igarashi, 1998. ${ }^{\mathrm{d}} \mathrm{Pandy}$ and Igarashi, 2000.

aged tap water with a density of $180-210$ larvae per container. The larvae were fed on a diet of yeast extract with mouse food powder and maintained at $25^{\circ} \mathrm{C}$. Adults were provided with $10 \%$ sucrose solution.

\section{Virus strains}

Virus isolates were originally obtained from the sera of patients in Nakhon Phanom Provincial hospital in Northeastern Thailand, during the dengue outbreak in 1993. The serotypes were determined as dengue 2 by reverse transcriptionpolymerase chain reaction (RT-PCR). Clinical information, serological response of the patients, in vitro infectivity and amino acid (AA) subtyping of coded strains used are shown in Table 2. Each of the virus isolates was obtained as a seed virus from the Department of Virology, Institute of Tropical Medicine, Nagasaki University.

\section{Virus assay}

A high titer of each isolate was prepared from the seed virus by inoculation into a monolayer culture of $A e$. albopictus clone C6/36 cell line and incubated at $28^{\circ} \mathrm{C}$ for 8 days in Eagle's medium supplement with $2 \%$ heat-inactivated fetal calf serum (FCS) and $0.2 \mathrm{~mm}$ each nonessential amino acids. The infected culture fluid was harvested 8 days after inoculation, aliquoted, and stored at $-80^{\circ} \mathrm{C}$ until used. Thus, the virus titer of the isolates was measured by the focus formation test by using BHK-21 cells on 96-well plates. Then three different titers were prepared by dilution with Eagle's medium supplement with $2 \%$ heat-inactivated fetal calf serum (FCS) and $0.2 \mathrm{~mm}$ each nonessential amino acids, and used for preparation of the infectious meal, blood virus sucrose solution (BVS).

\section{Oral infection}

To minimize age factors, only 4-5 dayold female mosquitoes were used. About 60 females were placed in cylindrical pint cardboard cages covered at one end with fine non-wettable nylon mesh. These females were deprived of food for 24-36 hrs. prior to the infectious meal. The females were allowed to feed with the infectious meal (BVS) consisting of equal volumes of isolate virus suspension, washed rabbit erythrocytes, and 10\% sucrose solution. Drops of the infectious meal were placed on the mesh covering the cardboard cage containing mosquitoes as previously described (Gubler et al., 1979). Feeding time was limited to 1-2 hrs, engorged mosquitoes were collected with an aspirator at $1 \mathrm{hr}$. intervals and transferred into clean cartons and maintained for up to 14 days at $30 \pm 1{ }^{\circ} \mathrm{C}$.

\section{Detection of virus infection and dissemina- tion}

Mosquito head and abdomen were severed and homogenized with a pellet mixer in a microtube containing Eagle's medium supplement with $2 \%$ heat-inactivated fetal calf serum (FCS) and $0.2 \mathrm{~mm}$ each nonessential amino acid. An aliquot of $150 \mu l$ of homogenized mosquito was clarified by centrifugation. The supernatant was filtrated through a $0.22 \mu \mathrm{m}$ filter and then inoculated into a monolayer culture of Ae. albopictus clone C6/36 cell line and incubated at $28^{\circ} \mathrm{C}$ for 8 days in Eagle's medium supplement with $2 \%$ heatinactivated fetal calf serum (FCS) and 0.2 mm each nonessential amino acids. After the culture fluid was harvested on the 8th day, the presence of the viral antigen was assayed by antigen detection-ELISA 
as described previously (Bundo-Morita, 1989). The detection of viral antigen in the homogenized abdomen was interpreted to indicate the mosquito midgut had become infected. Detection of viral antigen in the homogenized head indicated that the midgut had become infected and that the virus had subsequently disseminated to secondary target organs.

\section{Determination of Oral ID50}

The amount of virus to infect $50 \%$ of Ae. aegypti (oral ID50) was estimated as previously described (Gubler et al., 1979). Briefly, Ae. aegypti strains were fed on a suspension of virus strains of which titers were $4 \times 10^{4}, 4 \times 10^{3}$, and $2 \times 10^{2} \mathrm{FFU} / \mathrm{ml}$.
Then the percentage of mosquitoes infected was plotted against the virus titers of blood virus sucrose solution (BVS) used as the infectious meal.

\section{Statistical analysis}

The statistical analysis was done using Systat 7.0 software for Windows. A multiple comparison Chi-square analysis (with Yate's correction) was used to determine the significant difference among the infection and dissemination rates of the different isolate virus in each mosquito strain.

\section{RESULTS}

\section{Screening for susceptibility}

Significant variation in susceptibility

Table 3. Infection and Dissemination rates in Aedes aegypti from Nakhon Phanom (Thailand) orally infected with graded doses of two different isolates of dengue type- 2 virus.

\begin{tabular}{|c|c|c|c|c|}
\hline \multirow{3}{*}{ Titer of feeding suspension ${ }^{a}$} & \multicolumn{4}{|c|}{ Isolate virus strain } \\
\hline & \multicolumn{2}{|c|}{ ThNH7/93 } & \multicolumn{2}{|c|}{ ThNHpll/93 } \\
\hline & Infection $^{\mathrm{b}}$ & Dissemination $^{c}$ & Infection $^{\mathrm{b}}$ & Dissemination $^{c}$ \\
\hline $4 \times 10^{4} \mathrm{FFU} / \mathrm{ml}$ & $16 / 28(57)$ & $15 / 28(53)$ & $6 / 30(20)$ & $5 / 30(17)$ \\
\hline $4 \times 10^{3} \mathrm{FFU} / \mathrm{m} l$ & $13 / 33(39)$ & $12 / 33(36)$ & $4 / 24(17)$ & $3 / 24(13)$ \\
\hline $2 \times 10^{2} \mathrm{FFU} / \mathrm{ml}$ & $3 / 33(9)$ & $2 / 33(6)$ & $1 / 31(3)$ & $1 / 31(3)$ \\
\hline Total & $32 / 94(34)$ & $29 / 94(31)$ & $11 / 85(13)$ & $9 / 85(11)$ \\
\hline Oral ID50 ${ }^{\mathrm{d}}$ & \multicolumn{2}{|c|}{$3.51 \times 10^{4}$} & \multicolumn{2}{|c|}{$1.0 \times 10^{5}$} \\
\hline
\end{tabular}

${ }^{a}$ Determined by infectivity titration by focus formation in BHK-21 cells. ${ }^{b}$ Number of mosquitoes positive for dengue type-2 viral antigen in abdomen/number tested. ${ }^{\mathrm{c}}$ Number of mosquitoes positive for dengue type-2 viral antigen in head tissues/number tested. ${ }^{\mathrm{d}}$ Titer of isolate suspension required to infect $50 \%$ of mosquitoes $(\mathrm{FFU} / \mathrm{ml})$. The numbers in parentheses show percentage.

Table 4. Infection and Dissemination rates in Aedes aegypti from Escuintla (Guatemala) orally infected with graded doses of two different isolates of dengue type-2 virus.

\begin{tabular}{|c|c|c|c|c|}
\hline \multirow{3}{*}{ Titer of feeding suspension ${ }^{a}$} & \multicolumn{4}{|c|}{ Isolate virus strain } \\
\hline & \multicolumn{2}{|c|}{ ThNH7/93 } & \multicolumn{2}{|c|}{ ThNHpll/93 } \\
\hline & Infection $^{\mathrm{b}}$ & Dissemination $^{c}$ & Infection ${ }^{\mathrm{b}}$ & Dissemination $^{c}$ \\
\hline $4 \times 10^{4} \mathrm{FFU} / \mathrm{ml}$ & $5 / 44(11)$ & $5 / 44(11)$ & $5 / 30(17)$ & $4 / 30(13)$ \\
\hline $4 \times 10^{3} \mathrm{FFU} / \mathrm{ml}$ & $2 / 21(9)$ & $2 / 21(9)$ & $4 / 33(12)$ & $4 / 33(12)$ \\
\hline $2 \times 10^{2} \mathrm{FFU} / \mathrm{ml}$ & $2 / 35(6)$ & $1 / 35(3)$ & $1 / 23(4)$ & $1 / 23(4)$ \\
\hline Total & $9 / 100(9)$ & $8 / 100(8)$ & $10 / 86(12)$ & $9 / 86(10)$ \\
\hline Oral ID50 $0^{\mathrm{d}}$ & \multicolumn{2}{|c|}{$1.82 \times 10^{5}$} & \multicolumn{2}{|c|}{$1.18 \times 10^{5}$} \\
\hline
\end{tabular}

${ }^{\text {a}}$ Determined by infectivity titration by focus formation in BHK-21 cells. ${ }^{b}$ Number of mosquitoes positive for dengue type- 2 viral antigen in abdomen/number tested. "Number of mosquitoes positive for dengue type-2 viral antigen in head tissues/number tested. ${ }^{\mathrm{d}}$ Titer of isolate suspension required to infect $50 \%$ of mosquitoes $(\mathrm{FFU} / \mathrm{ml})$. The numbers in parentheses show percentage. 
for the oral infection with the two isolates was observed. The overall infection rate for the two isolate virus in mosquitoes from Thailand ranged from 12.9-34.0\% (Table 3), and $9.0-11.6 \%$ in mosquitoes from Guatemala (Table 4). The Ae. aegypti strain from Thailand (Nakhon Phanom) showed the highest susceptibility, and was more susceptible to the isolate $\mathrm{ThNH}$ $7 / 93$ (DSS) than to the isolate ThNHpll/93 (DF), $P<0.05, \chi^{2}=13.283$, df $=1$; whereas the $A e$ aegypti strain from Guatemala (Escuintla) had a slightly lower infection rate, and a significant variation in susceptibility for the oral infection was not observed with either of the two isolate viruses used, $P>0.05, \chi^{2}=0.049, \mathrm{df}=1$. The results demonstrate that the mosquito strain from Thailand is more susceptible to the oral infection with either isolate virus strain than the mosquito strain from Guatemala.

\section{Ascertaining the infectivity}

Variation in the infectivity rate between Ae. aegypti strains was observed for the virus strains. For the isolate ThNH7/93 (DSS), infection and dissemination rates ranged from 9-34\% and 8-32\%, respectively; and for the isolate ThNHpll/93 (DF) ranged from $11-13$ and $10-11 \%$, respectively (Tables 3 and 4). Infection and dissemination rates of the isolate ThNH7/ 93 (DSS) were significantly higher in the Ae. aegypti strain from Thailand (Nakhon Phanom) than those in the strain from Guatemala (Escuintla), $P<0.05, \chi^{2}=6.833$, $\mathrm{df}=1$; whereas for the isolate ThNHpll/93 (DF), even though slightly higher in the Ae. aegypti strain from Thailand than those in the strain from Guatemala, the observed rates were not significantly different, $P>0.05, \chi^{2}=0.557, \mathrm{df}=1$. Furthermore, in a parallel experiment at 5 th day of post-infection the ThNH7/93 (DSS) showed an infection and dissemination rate significantly higher than that of ThNHpll/93 (DF) in either Ae. aegypti strain, $P<0.05, \chi^{2}=9.167, \mathrm{df}=1$ (data not shown). On the other hand, when the amount of each isolate virus to infect orally 50\% of mosquitoes (oral ID50) was determined for each mosquito strain as described in Materials and Methods, the results showed that oral ID50 $(\mathrm{FFU} / \mathrm{ml})$ of ThNH7/93 and ThNHpll/93 for Ae. aeg$y p t i$ from Thailand were $3.51 \times 10^{4}$ and 1.0 $\times 10^{5}$ respectively (Table 3 ); whereas oral ID50 of ThNH7/93 and ThNHpll/93 for Ae. aegypti from Guatemala were $1.82 \times 10^{5}$ and $1.18 \times 10^{5}$ respectively (Tables 3 and 4).

\section{Discussion}

Variability in susceptibility to experimental oral infection with all 4 serotypes of dengue virus is well known for Aedes sp. and strains of a given species (Tesh et al., 1976; Aitken et al., 1977; Grimstand et al., 1977; Tardieux et al., 1990). Despite all these observations, the susceptibility, infection and dissemination rate in orally infected $A e$. aegypti originating from different geographic localities with dengue virus isolated from patients exhibiting different disease severity has not been described yet. Therefore, in the present study the capacity of two different isolates of dengue type- 2 virus to infect and disseminate in orally infected $A e$ aegypti strains was ascertained.

The present study on the isolate viruses ThNH7/93 and ThNHpll/93 which were isolated from patients with clinical manifestation of dengue shock syndrome (DSS) and classical dengue fever (DF), respectively, has shown that significant variation in susceptibility to oral infection with virus isolates occurred between strains of Ae. aegypti originating from different geographic localities. Even though the infection rate for the two isolate virus strains in the Ae. aegypti strain from Guatemala was lower than that in the strain from Thailand (Tables 3 and 4), the observed infection rates are consistent with previous works that have reported infection rates for dengue type-2 virus of $3-57 \%$ (Tesh et al., 1976; Grimstand et al., 1977; 
Tardieux et al., 1990). The results might suggest that the susceptibility patterns to the oral infection with the two isolate viruses observed were similar, suggesting that the factors controlling susceptibility to oral infection in the Ae. aegypti strains tested could be the same for both isolate viruses. It is noteworthy that the $A e$ a $a$ gypti strain from the area where the isolate viruses were isolated (Table 1) showed to be significantly more susceptible to the isolate virus ThNH7/93 than the isolate virus ThNHpll/93, which might suggest that the trend was toward higher susceptibility to the virus isolated from a patient with severe clinical manifestation (DSS). Therefore, even though the Ae. aegypti strain from Guatemala did not show a significant difference in susceptibility to oral infection for the two isolate viruses, similar susceptibility mode indicated in the Ae. aegypti strain from Thailand is likely to occur in Ae. aegypti in Guatemala if the dengue type- 2 virus of Southeast Asian genotype happens to invade into the country.

Mangada and Igarashi (1998) in their study on biological in vitro properties reported that the ThNH7/93 isolate produced significantly larger plaques than the ThNHpll/93. It was speculated that the difference might be due to certain genetic elements of the isolate virus. Furthermore, Pandy and Igarashi (2000) classified the isolates ThNH7/93 and ThNHpll/93 into amino acid (AA) subtype I and III, respectively, and reported a possible correlation between the amino acid sequence subtype of the virus strain and clinical severity of the patient from whom each virus strain was isolated. In the present study, infection and dissemination rate for the isolates ThNH7/93 and ThNHpll/93 were not significantly different in $A e$. aeg$y p t i$ from Guatemala. However, in Ae. aeg$y p t i$ from Thailand it was observed that the isolate ThNH7/93 seemed to be more infective than the isolate ThNHpll/93, which correlated with severity of disease exhibited by the patients from whom each isolate virus strain was isolated. As in the studies previously mentioned (Mangada and Igarashi, 1998; Pandy and Igarashi, 2000), the differences in susceptibility, infection and dissemination rates of both isolate viruses between the Ae. aegypti strains observed in the present study may be due to molecular elements of the isolate virus.

In Guatemala, even though DSS cases have not been reported up to the present time, apparently are being experienced similar continued cycles of $\mathrm{DH} / \mathrm{DHF}$ epidemics, as previously observed in countries in Southeast Asia (PAHO, 2000). Our findings showed that the Ae. aegypti strain originated from Guatemala (Escuintla) was susceptible to oral infection with the virus isolated from patients originated from a Southeast Asian country exhibiting either DSS or DF symptoms. Thus, eventual introduction of the dengue type2 virus Southeast Asian genotype, which is associated with severe clinical manifestations, and subsequently possible DSS manifestations, should be taken into consideration as a potential risk factor in Guatemala.

\section{ACKNOWLEDGMENTS}

The authors acknowledge the technical assistance of M. Nagata and E. Urakawa. We also thank M. Shimada for statistical support and N. Phumala and M. Pascucci for critically reading the manuscript. The first author is a recipient of a Mombusho scholarship from the Ministry of Education, Science, Sports and Culture of Japan for his stay and study in the Institute of Tropical Medicine, Nagasaki University.

\section{REFERENCES}

Aitken, T. H. G., Downs, W. G. and Shope, R. E. 1977. Aedes aegypti strain fitness for yellow fever virus transmission. Am. J. Trop. Med. Hyg., 26: 985-989.

Boromisa, R. D., Rai, K. S. and Grimstad, P. R. 1987. Variation in the vector competence of geographic strains of Aedes albopictus for dengue 1 virus. $J$. 
Am. Mosq. Control. Assoc., 3: 378-386.

Bundo-Morita, K. 1989. Detection of Japanese Encephalitis virus antigens by the sandwich ELISA in infected cell culture fluid and cell homogenates. Trop. Med., 31: 49-65.

Grimstad, P. R., Craig, G. B., Jr, Ross, Q. E. and Yuill, T. M. 1977. Aedes triseriatus and La Crosse virus: geographic variation in vector susceptibility and ability to transmit. Am. J. Trop. Med. Hyg., 26: 990996.

Gubler, D. J. and Clarck, G. G. 1995. Dengue/dengue haemorrhagic fever, the emergence of a global health problem. Emerg. Infect. Dis., 1: 55-57.

Gubler, D. J., Nalim, S., Tan, R., Saipan, H. and Saroso, J. S. 1979. Variation in susceptibility to oral infection with dengue viruses among geographic strains of Aedes aegypti. Am. J. Trop. Med. Hyg., 28: 1045-1052.

Gubler, D. J. and Rosen, L. 1976. Variation among geographic strains of Aedes albopictus in susceptibility to infection with dengue viruses. Am. J. Trop. Med. Hyg., 25: 318-325.

Mangada, M. N. and Igarashi, A. 1998. Molecular and in vitro analysis of eight dengue type 2 viruses isolated from patients exhibiting different disease severities. Virology, 244: 458-466.

Monath, T.P. 1994. Yellow fever and dengue: the interactions of virus, vector and host in the reemergence of epidemic disease. Semin. Virol., 5: 133-145.

Monath, T. P. and Heinz, F. X. 1996. Flaviviruses. In: Virology 3rd ed., (ed. Fields, B. N., Knipe, D. M.,
Howeley, P. M. et al.), pp. 961-1034, LippincottRaven, Philadelphia.

Morens, D. M., Marchete, N. J., Chu, M. C. and Halstead, S. B. 1991. Growth of Dengue Type 2 virus isolates in human peripheral blood leukocytes correlates with severe and mild dengue disease. $A m$. J. Trop. Med. Hyg., 45: 644-651.

Pan American Health Organrization (PAHO) 2000. Health situation analysis in the Americas, 19992000, Dengue in Central America: The epidemics of 2000. Epidemiol. Bull., 21: 4-8.

Pandy, B. D. and Igarashi, A. 2000. Severity-related molecular differences among nineteen strains of dengue type 2 viruses. Microbiol. Immunol., 44: 179-188.

Rosen, L., Roseboom, L. E., Gubler, D. J., Lien, J. C. and Chaniotis, B.C. 1985 . Comparative susceptibility of mosquito species and strains to oral and parenteral infection with dengue and Japanese encephalitis viruses. Am. J. Trop. Med. Hyg., 34: 603-615.

Tardieux, I., Poupel, O., Lapchin, L. and Rodhain, F. 1990. Variation among strains of Aedes aegypti in susceptibility to oral infection with dengue virus type 2. Am. J. Trop. Med. Hyg., 43: 308-313.

Tesh, R. B., Gubler, D. J. and Rosen, L. 1976. Variation among geographic strains of Aedes albopictus in susceptibility to infection with chikungunya virus. Am. J. Trop. Med. Hyg., 25: 326-335.

World Health Organization (WHO) 2000. Dengue/ dengue haemorrhagic fever. weekly epidemio logical record, 24: 193-196. 